\title{
A low cost route optimization scheme for cluster-based proxy MIPv6 protocol
}

\begin{abstract}
Proxy Mobile IPv6 (PMIPv6) is a network based mobility protocol which has been designed to relieve the mobile nodes (MNs) from participating in the mobility process and to reduce the long handoff latency of the MIPv6 protocol. However, PMIPv6 incurs a long communication path due to the triangle routing problem, in which, all packets sent by MNs are obligated to pass through the local mobility anchor. Several solutions have been proposed to mitigate this issue. However, they still incur high signaling overhead to recover the Route Optimization (RO) status after handoff. In this paper, we propose a Cluster-Based RO (CBRO) scheme for the clustered architecture of the PMIPv6, in which, the Mobile Access Gateways (MAGs) are grouped into clusters with a distinguished Head MAG (HMAG) for each. In the proposed CBRO, the RO process is relied on the HMAGs to reduce the handoff latency while achieving a fast recovery of the optimized path after handoff. The proposed CBRO is evaluated analytically and compared with the basic PMIP and the current RO schemes. The obtained numerical results have shown that the proposed CBRO outperforms all other schemes in terms of signaling cost required to recover the RO status after handoff and the total cost performance metrics.
\end{abstract}

Keyword: Cluster-based PMIPv6; Handoff; PMIPv6; Route optimization 